\title{
Diacronie
}

Studi di Storia Contemporanea

$N^{\circ} 10,2 \mid 2012$

Digital History: la storia nell'era dell'accesso

\section{Vecchie abitudini e nuovi strumenti}

Un sistema informativo per lo studio delle vicende architettoniche del Palazzo Ducale di Mantova

\section{Lisa Valli}

\section{Q OpenEdition}

Journals

\section{Edizione digitale}

URL: http://journals.openedition.org/diacronie/2860

DOI: 10.4000/diacronie.2860

ISSN: 2038-0925

Editore

Association culturelle Diacronie

Notizia bibliografica digitale

Lisa Valli, «Vecchie abitudini e nuovi strumenti », Diacronie [Online], N10, 2 | 2012, documento 7,

Messo online il 29 juin 2012, consultato il 01 mai 2019. URL : http://journals.openedition.org/ diacronie/2860 ; DOI : 10.4000/diacronie.2860 


\section{Diacronie}

\section{$7 /$}

\section{Vecchie abitudini e nuovi strumenti}

\section{Un sistema informativo per lo studio delle vicende architettoniche del Palazzo Ducale di Mantova}

Lisa VALLI*

Il contributo offre una prima panoramica sulla possibile interazione fra i più tradizionali metodi di ricognizione delle fonti archivistiche e di descrizione dei beni architettonici (ad esempio il Raumbuch) e gli strumenti della digital history, in riferimento al settore della ricerca sui beni culturali, e specificamente sui beni architettonici. Si espone l'impiego di questo approccio nella gestione, referenziazione e trasmissibilità dei dati raccolti nel percorso di ricerca relativo alla ricognizione delle fonti per la storia del complesso del Palazzo Ducale di Mantova dal XIX secolo ad oggi.

\section{Introduzione}

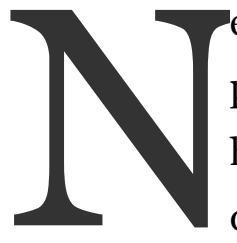
el settore della ricerca inerente la conservazione dei beni culturali, ed in particolare dei beni architettonici, gli strumenti della digital history hanno oggi un ruolo chiave nell'economia di gestione e referenziazione dei

dati raccolti, così come nella trasmissibilità - ed accertabilità - della ricerca stessa. Un bene architettonico presenta una connaturata molteplicità di aspetti da prendere in analisi nel caso si voglia dare testimonianza delle sue caratteristiche morfologiche, spaziali e materiche; ancora di più se si cerchi di comprenderne le logiche costruttive, le dinamiche di impiego e quelle dell'eventuale trasformazione. Il percorso di ricerca - di cui il sistema informativo che verrà di seguito presentato costituisce uno dei principali strumenti - mira a ricostruire le vicende storiche architettoniche del Palazzo Ducale di Mantova nei secoli XIX e XX. Il complesso architettonico identificato come Palazzo Ducale è uno dei più grandi complessi di edifici monumentali di origine medievale sino ad oggi preservatosi. La sua costruzione, 
che prese l'avvio almeno nel XIII secolo, trova un assetto pressoché definitivo sul finire del Settecento, mentre trasformazioni conseguenti a cambi di destinazione d'uso e ad interventi più o meno conservativi, si sono susseguite fino ad oggi. Il complesso, per come odiernamente si presenta, è composto nella parte "monumentale" da 902 ambienti censiti ${ }^{1}$, ed articolato secondo caratteristiche più propriamente urbane che architettoniche: non un edificio, quindi, ma un insieme eterogeneo di costruzioni e piazze, strade e giardini. In assenza di adeguati sistemi informativi sul bene sussiste una concreta difficoltà di identificazione e caratterizzazione degli ambienti, premessa imprescindibile alle attività di gestione e conservazione. La conseguenza diretta è una impossibilità di diretta referenziazione dei dati estratti dalle fonti documentarie agli ambienti, possibile solo per gli ambienti principali e maggiormente conosciuti. Le fonti documentarie moderne e contemporanee per il Palazzo sono altresì copiosissime, ma tanto frammentate quanto lo erano gli interessi sul Palazzo da parte di differenti enti ed istituzioni. Tali fonti, oltre ad essere conservate in numerosi fondi archivistici, sono rinvenibili in altrettanti archivi sia italiani che esteri, sia archivi pubblici che privati.

\section{A cosa serve un sistema informativo?}

La necessità di redigere ed impiegare un sistema informativo come strumento base per la ricerca storica sui beni culturali presuppone una serie di considerazioni delle quali si cerca di dare brevemente conto. La creazione di un sistema informativo è una possibile risposta alla necessità di soddisfare esigenze di condivisione, verifica e trasmissibilità di un insieme di operazioni che portino alla creazione di beni materiali o immateriali ${ }^{2}$. Il sistema informativo scaturisce dalla sinergia delle risorse economiche, umane, tecniche e tecnologiche che cooperano al fine di raccogliere, organizzare, comunicare e permettere la ricerca e la trasmissione di dati generati da un'attività di un ente o di una organizzazione ${ }^{3}$. La scelta di creare un sistema informativo sarà di conseguenza tanto più appropriata quanto il sistema sarà in grado di soddisfare le necessità espresse dall'ente che lo genera, di rispecchiarne la logica operativa e di essere intelligibile da soggetti esterni all'ente. È tuttavia la sostanziale differenza che

${ }^{1}$ Oltre ai 902 ambienti censiti esistono circa un centinaio di ambienti non ancora censiti, a cui si deve aggiungere l'ulteriore porzione del Palazzo oggi non più accorpata a quella monumentale. ${ }^{2} \mathrm{Si}$ consiglia per approfondimenti sul tema un confronto con il contributo di VALENTI, Filippo, Nozioni di base per un'archivistica come euristica delle fonti documentarie, in GRANA, Daniela (a cura di), Scritti e lezioni di archivistica, diplomatica e storia istituzionale, Roma, Pubblicazioni degli Archivi di Stato, 2000, pp. 135-224.

3 VICO, Franco, Costruire il GIS: dati versus processi, in GASTALDO, Guido, PANZERI, Matteo (a cura di), Sistemi Informativi Geografici e Beni Culturali, Torino, Celid, 2000. 
intercorre fra i concetti di dato e di informazione a giustificare definitivamente l'esigenza della redazione di sistemi informativi necessari per via del sussistere di basi di dati non diversamente governabili. Il dato è un elemento di conoscenza, ma privo di contesto; i dati costituisco gli elementi alla base di una informazione4. L’informazione è altresì un elemento conoscitivo, ma deriva necessariamente dalla contestualizzazione di un dato, quindi dall'individuazione delle relazioni che esistono all'interno di un insieme di dati5. Un sistema informativo è, pertanto, un sistema di organizzazione e gestione di dati funzionali all'ottenimento di informazioni necessarie alle attività di un ente, la cui efficienza - è sempre bene ribadire - è assolutamente indipendente dal suo grado di automazione. Un sistema informativo che preveda la possibilità di compiere elaborazioni automatiche di dati al fine di ricavarne evidenze necessita di una componente informatica, generando una partizione interna al nostro sistema che può essere definito come sistema informativo informatico ${ }^{6}$. Un sistema informativo informatico si basa sul passaggio da una strumentazione analogica ad una digitale. Si serve del computer, quindi uno strumento il cui funzionamento è basato su un sistema binario: il sistema informativo informatico è un apparato che elabora informazioni, le traduce, le trasforma, le digitalizza attraverso un sistema binario e necessita di una memoria attraverso cui immagazzinare l'apparato di dati alla base delle sue elaborazioni.

Nel nostro caso abbiamo due principali categorie di dati, gli ambienti del palazzo di cui vogliamo indagare le vicende architettoniche e le fonti documentarie che le documentano.

SISTEMA RELAZIONARE

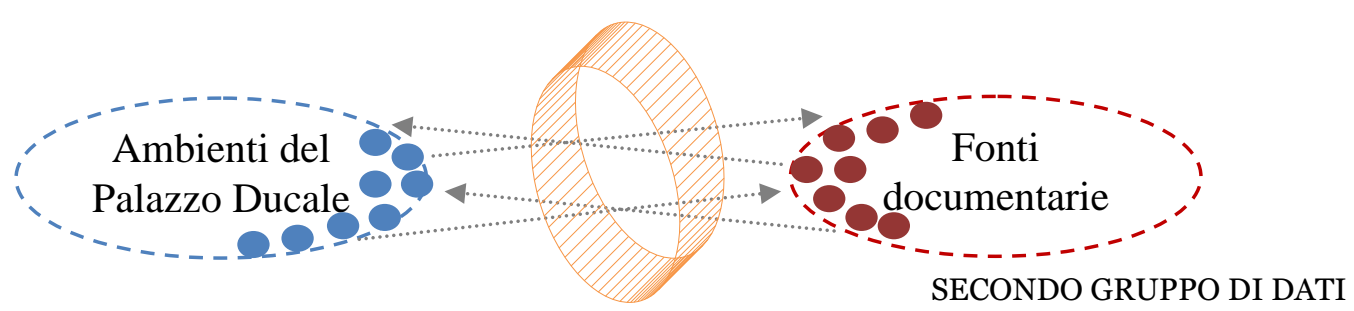

PRIMO GRUPPO DI DATI

Un sistema informativo è costituito da uno schema concettuale, un processo concettuale ed una base informativa. La base informativa è la memoria del nostro

\footnotetext{
4 CARUCCI, Paola, Introduzione generale, in PIANO MORTARI, Maria Teresa, SCANDALIATO CICANI, Isotta (a cura di), Le fonti archivistiche. Catalogo delle guide e degli inventari editi (1861-1991), Roma, Ufficio Centrale per i Beni Archivistici, 1995, pp. 7-23.

5 ATZENI, Paolo, CERI, Stefano, PARABOSCHI, Stefano, TORLONE, Riccardo, Basi di dati. Modelli e linguaggi di interrogazione, Milano, McGraw-Hill, 2002.

6 GUERCIO, Maria, Archivistica informatica, Roma, Carocci, 2001.
} 
sistema che contiene i dati; è la componente variabile del nostro sistema poiché, se correttamente impostata - dal momento che è sempre implementabile essendone lo schema concettuale - è la "pianta organica" in cui i dati si collocano ed individua le sottocategorie in cui dati possono essere suddivisi. Il processo concettuale è l'elemento dinamico del sistema: permette ai dati di essere associati alla corrispondente collocazione costituendo la componente stabile del sistema7. Il sistema informativo di seguito presentato è quindi concepito come strumento ausiliario alla conoscenza del bene, di documentazione del censimento delle fonti e principalmente come strumento relazionare fra le fonti ed il bene stesso. I differenti elementi che compongono il sistema informativo sono stati creati supportando sia il censimento delle fonti che la formazione della base di dati attraverso l'impiego di softwares opensource relativamente semplici e ben diffusi - principalmente quelli offerti da OpenOffice.org al fine di garantire la più estesa accessibilità sia economica che tecnica ai dati raccolti ed alle informazioni derivate dal percorso di ricerca.

\section{La problematica dell'identificazione univoca degli ambienti}

Oggetto dello studio è il Palazzo Ducale di Mantova, un complesso architettonico monumentale costituito da circa un migliaio di ambienti, di cui si vogliono ricostruire, in questa prima fase, le vicende storiche del complesso a partire dalla formazione del suo primo nucleo, risalente alla prima metà del secolo XIV. La principale problematica facilmente riscontrabile ed immediatamente emersa all'avvio del percorso di ricerca è direttamente connessa alla natura stessa dell'oggetto dello studio: l'identificazione univoca dei singoli ambienti e la corretta referenziazione a questi dei dati reperiti. La lunga storia del complesso e il continuo avvicendamento di differenti utilizzatori hanno comportato continue trasformazioni, alle quali hanno necessariamente fatto seguito altrettante mutazioni identificative degli edifici, degli appartamenti e degli ambienti. Per tali ragioni l'identificazione di un ambiente attraverso un toponimo ha ragion d'essere solo se riferita alla datazione cronologica del toponimo stesso. Di fronte all'estensione del complesso ed all'eterogeneità dei soggetti coinvolti a vario titolo nello studio, nella gestione e nella fruizione attuale della struttura, le reali possibilità di identificare univocamente un ambiente facendo unicamente riferimento ai toponimi risulta spesso fallimentare. Tale problematica non è certo emersa ora: si consideri, a titolo di esempio, come nei documenti settecenteschi relativi alla gestione del Palazzo,

7 D’AMICO, Giuseppe, Progettazione concettuale di sistemi informativi, Milano, Hoepli, 1990. 
agli elenchi di chiavi fossero state fatte corrispondere lunghe descrizioni per permettere l'identificazione degli ambienti. Negli ultimi anni nel Palazzo Ducale è stato introdotto da parte della direzione del museo un sistema di classificazione univoco degli ambienti che li identifica attraverso la segnalazione del quartiere, del livello di appartenenza e di una numerazione progressiva attribuita all'ambiente stesso. Tale numerazione è parziale poiché tiene conto dei soli ambienti immediatamente disponibili del museo e degli uffici insediati nel complesso, mentre non interviene sugli ambienti diversamente destinati che sono, tuttavia, parte integrante del complesso. Il sistema di codifica non è strettamente alfanumerico, poiché prevede, oltre all'impiego di lettere e numeri, anche l'utilizzo di simboli, fattore che implica l'impossibilità di un impiego diretto dei codici quali chiavi di riconoscimento per l'eventuale redazione di un sistema informativo territoriale in cui referenziare i dati raccolti sul complesso.

\section{L'adozione del sistema di codifica del Raumbuch, con alcuni accorgimenti}

Fra i differenti metodi di approccio all'analisi di un edificio sviluppati negli ultimi decenni, quello che sembra meglio prestarsi all'analisi dei grandi complessi e meglio rispondere all'esigenza di documentazione di stratificazioni storiche complesse è il Raumbuch. Il metodo di redazione del Raumbuch, tradotto in ambito italiano con la locuzione libro delle stanze, viene diffuso al di fuori dell'ambito tedesco, dove seppur non formalizzato, era già stato ampiamente sperimentato, agli inizi degli anni Novanta del secolo ventesimo a seguito della pubblicazione della Praktische Denkmalpflege ${ }^{8}$ di Mader e Petzet. L'opera nel suo complesso si pone come raccolta ordinata di suggerimenti con cui affrontare l'analisi di un edificio, operando attraverso un metodo scientificamente corretto e prevedendo il dispendio di risorse limitate: in sostanza si tratta un sistema economico in termini di costi, tempi ed efficienza per l'approccio al manufatto. L'edificio viene identificato nel suo insieme, si individuano i livelli su cui gli ambienti si articolano e, infine, gli ambienti stessi. Compiuta l'identificazione dell'ambiente si procede alla scomposizione virtuale dello stesso nelle sue sei facce principali: soffitto, pavimento e le quattro pareti, sulle quali si vanno ad identificare tutti gli elementi presenti - dalle aperture alle stratificazioni degli intonaci descrivendo successivamente attraverso specifiche schede i singoli elementi nelle loro

\footnotetext{
8 PETZET, Michael, MADER, Gert, Praktische Denkmalpflege, Monaco, Kohlhammer, 1993. 
caratteristiche materiali, tecniche e conservative9. Di questo articolato sistema di procedure ci interessa, nell'ottica di predisporre le basi per la redazione del Raumbuch del Palazzo, affrontare il sistema di codifica che propone; questa operazione è stata avviata da due anni per mezzo delle esercitazioni compiute dagli studenti dei corsi di Restauro architettonico della Facoltà di Architettura della sede di Mantova del Politecnico di Milano sotto la guida del prof. Alberto Grimoldi. Già dai primi risultati emersi le potenzialità del sistema del Raumbuch applicate allo studio del Palazzo non sembrano affatto disattese e se ne auspica di conseguenza la prosecuzione. Il sistema è non solo "contenitore strutturato" di dati eterogenei sia per contenuto che per tipologia - come già segnalato dagli autori di interventi sulla questione - ma anche efficiente strumento identificativo, e questo aspetto è quello che si va ora ad analizzare.

Un "codice tipo" che possiamo riscontrare su un quaderno di Raumbuch può essere ad esempio "03 - 04 • c . 247".

Il codice "livello" indica il piano a cui l'ambiente è collocato e convenzionalmente al livello 01 si fa corrispondere il piano interrato, al livello 02 si fa corrispondere il piano terreno e così via, a salire, fino a quando si sia dato conto di tutti i livelli.

Il codice "ambiente" identifica l'ambiente in analisi, numerato progressivamente, normalmente partendo dall'ambiente a cui si ha accesso e poi, gradualmente, avanzando negli ambienti sullo stesso livello. Le superfici di un singolo ambiente vengono poi identificate generalmente dal codice "superficie" attraverso l'impiego di lettere in alfabeto minuscolo, attribuendo convenzionalmente la lettera "a" al soffitto, la lettera "b" al pavimento e poi, a seguire, le pareti secondo la rotazione "est - sud ovest - nord”. Il codice "unità" dà conto dell'unità dell'oggetto di analisi - in genere sulla base di una classificazione merceologica - secondo la quale si attribuiscono convenzionalmente i numeri da 100 a 199 ad intonaci e superfici, i numeri da 200 a 299 ad aperture e serramenti, i numeri da 300 a 399 agli elementi di impiantistica; i numeri a seguire sono attribuiti ad altre tipologie di unità.

\footnotetext{
${ }^{9} \mathrm{Si}$ veda in merito all'adozione del metodo nell'ambito italiano la tesi di laurea di BALBONI, Laura, CORRADINI, Paolo, Rilievo, diagnosi e monitoraggio del degrado statico della "Trostburg" a Waidbruck (BZ), Tesi di laurea, relatore prof. Alberto Grimoldi, Politecnico di Milano, Facoltà di Architettura, Campus Leonardo, Sede di Mantova, a.a. 2003/2004, così come il contributo di DEL CURTO, Davide, Das Raumbuch zwischen Bauforschung und Schadenskartierung: Zeiträume und Ursachen für Schäden von Burg Trostburg bei Waidbruck bis Mantua, in DIEKAMP, Anja (Hrsg.), Naturwissenschaft und Denkmalpflege, Innsbruck, Innsbruck University Press, 2007, pp. 79-90.
} 


\section{Il sistema di codifica proposto e gli strumenti grafici di supporto impiegati}

Adottando la logica di codifica proposta dal metodo del Raumbuch, si è cercato di mantenere la maggior continuità possibile con la

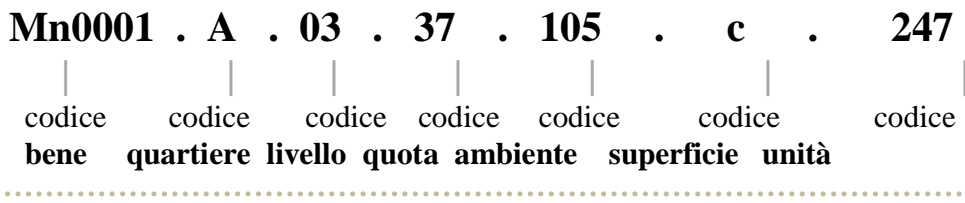
classificazione

introdotta dalla direzione del museo. Salvaguardando la caratteristica di sistema aperto, si sono introdotti fattori di modifica al solo fine di permettere un'implementazione delle informazioni contenute nella stringa identificativa, cercando di ridurre il più possibile la ridondanza di informazioni e le possibilità di equivoco ed impiegando unicamente caratteri alfanumerici per le ragioni sopra esposte. Un "codice identificativo tipo" proposto si presenta secondo la scansione "MNooo1 . A . 03 37 . 105 . c . 247", questo può ad esempio identificare in modo univoco una finestra che sarà solo ed esclusivamente quella a cui il codice fa riferimento in tutto il complesso architettonico e non vi sarà un altro elemento a cui sia associato lo stesso codice.

Il complesso del Palazzo Ducale di Mantova è stato identificato come "MNooo1". MN fa riferimento alla sigla identificativa della città di Mantova, mentre "ooo1” è l'identificativo per il Palazzo Ducale, identificazione propiziatoria scelta nella speranza che tale sistema informativo possa rivelarsi utile all'indagine in altri cantieri. All'interno degli elaborati prodotti riferiti al bene in analisi si è riportato per brevità la parte di codice segnalata nel riquadro sopra "A . $03 \cdot 37 \cdot 105$ " poiché sono stati redatti allo scopo di identificare gli ambienti codificati sulle planimetrie.

Divisione in quartieri. Il complesso del Palazzo è stato diviso dalla codifica proposta dalla direzione in quartieri, che vanno ad identificare, secondo le denominazioni maggiormente durature, la divisione storicamente assunta nel tempo dai gruppi di edifici.

\begin{tabular}{|c|c|c|c|c|c|c|c|}
\hline \multicolumn{2}{|c|}{$\begin{array}{l}\text { A_QUARTIERE DI } \\
\text { CASTELLO }\end{array}$} & \multicolumn{2}{|c|}{$\begin{array}{l}\text { B_QUARTIERE DI CORTE } \\
\text { VECCHIA }\end{array}$} & \multicolumn{2}{|c|}{$\begin{array}{l}\text { C_QUARTIERE DELLA } \\
\text { DOMUS NOVA }\end{array}$} & \multicolumn{2}{|c|}{$\begin{array}{l}\text { D_QUARTIERE DI CORTE } \\
\text { NUUOVA }\end{array}$} \\
\hline $\mathrm{N}^{\circ}$ LIVELLI & 5 & $\mathrm{~N}^{\circ}$ LIVELLI & 4 & NoLIVELLI & 6 & NoLIVELLI & 3 \\
\hline $\mathrm{N}^{\circ} \mathrm{AMBIENTI}$ & 178 & $\mathrm{~N}^{\circ} \mathrm{AMBIENTI}$ & 261 & $\mathrm{~N}^{\circ} \mathrm{AMBIENTI}$ & 224 & $\mathrm{~N}^{\circ} \mathrm{AMBIENTI}$ & 224 \\
\hline \multicolumn{2}{|c|}{ A_0_22_N ${ }^{\circ}$ AMBIENTI_24 } & \multicolumn{2}{|c|}{ B_0_22_NAMBIENTI_8 } & \multicolumn{2}{|c|}{ C_0_21_N ${ }^{\circ}$ AMBIENTI_26 } & \multicolumn{2}{|c|}{ B_0_21_N ${ }^{\circ}$ AMBIENTI_8 } \\
\hline \multicolumn{2}{|c|}{ A_0_24_N ${ }^{\circ}$ AMBIENTI_48 } & \multicolumn{2}{|c|}{ B_0_25_N N AMBIENTI_136 } & \multicolumn{2}{|c|}{ C_0_24_N ${ }^{\circ}$ AMBIENTI_73 } & \multicolumn{2}{|c|}{ B_0_24_N ${ }^{\circ}$ AMBIENTI_136 } \\
\hline \multicolumn{2}{|c|}{ A_1_27_N ${ }^{\circ}$ AMBIENTI_62 } & \multicolumn{2}{|c|}{ B_1_28_NºMBIENTI_84 } & \multicolumn{2}{|c|}{ C_1_27_NAMBIENTI_67 } & \multicolumn{2}{|c|}{ B_1_28_NAMBIENTI_84 } \\
\hline \multicolumn{2}{|c|}{ A_2_31_NºMBIENTI_39 } & \multicolumn{2}{|c|}{ B_2_34_N'AMBIENTI_33 } & \multicolumn{2}{|c|}{ C_2_32_N ${ }^{\circ}$ AMBIENTI_26 } & \multicolumn{2}{|c|}{ B_2_33_N ${ }^{\circ}$ AMBIENTI_33 } \\
\hline \multicolumn{2}{|c|}{ A_3_33_N'AMBIENTI_5 } & & & \multicolumn{2}{|c|}{ C_3_35_N ${ }^{\circ}$ AMBIENTI_22 } & & \\
\hline & & & & \multicolumn{2}{|c|}{ C_4_N'AMBIENTI_10 } & & \\
\hline
\end{tabular}


Per continuità con il sistema adottato è stata mantenuta questa suddivisione alla quale andiamo ad addizionare un ulteriore quartiere, che definiremo "E - Edifici esterni alla parte monumentale" e che comprende le fabbriche originariamente comprese nel complesso del Palazzo, ma ora non più comprese nella cosiddetta parte monumentale.

Divisione in livelli. L'adozione di questa ripartizione rende però difficilmente intelligibile la divisione in livelli proposta dalla direzione così come dallo schema tradizionale di Raumbuch. Poiché le quote corrispondenti ad esempio al "livello 1" per un edificio non corrispondono al medesimo livello per un altro edificio limitrofo - ed ancora meno se si confrontano fra loro i quartieri - non si riesce a compiere raffronti sulla localizzazione verticale degli ambienti. Si è pertanto deciso di integrare questo metodo. Il più recente rilievo completo della parte monumentale del Palazzo Ducale oggi disponibile è quello compiuto nel 1993 dal Ministero per i lavori pubblici, Magistrato alle Acque, Provveditorato generale alle opere pubbliche, Nucleo operativo di Mantova, sotto la direzione dell'ingegnere G. Quarta. Seppure tale rilievo sia stato compiuto per dare conto delle geometrie complessive della struttura e dei suoi ambienti e sia risultato indubbiamente necessario, non è tuttavia sufficiente ad un'analisi approfondita della complesso a fini conservativi. Di questo rilievo sembra utile adottare il metodo di identificazione delle sezioni orizzontali, che vengono identificate attraverso la quota verticale assoluta rispetto al livello del mare. Se andiamo quindi a segnalare che un ambiente si colloca, ad esempio, al livello "o3" - associando a questo il valore "37" metri sul livello del mare - osserviamo come a tale quota sia possibile compiere una rappresentazione della pianta dell'ambiente ragionevolmente esaustiva: le aperture principali sono rappresentabili a tale quota poiché ne intercettano il piano virtuale di sezione; il piano di calpestio sarà collocato un metro al di sotto circa di tale quota, da cui si ha accesso all'ambiente. Questo permette inoltre di poter confrontare la collocazione degli ambienti tra gli edifici nei differenti quartieri, grazie ad un riferimento assoluto esterno al sistema di classificazione adottato. Questa scelta non vieta, a lato della codifica, di dare conto anche della corresponsione fra la quota del livello e la funzione che questo assume nei contesti dei singoli edifici. Per i livelli successivi della codifica si fa riferimento a quanto proposto dal metodo del Raumbuch sopra introdotto. I codici sono stati riportati - in collaborazione con il dottor Michele Cassini -in formato vettoriale ( $\mathrm{dwg}$ ) sulle planimetrie in modo da poter essere impiegati come immediato strumento di lavoro e predisposti in un formato compatibile ai più diffusi sistemi Gis/Sit. 


\section{La complessità delle fonti documentarie}

Come segnalato nell'introduzione, le fonti documentarie relative alla storia ottocentesca e novecentesca del Palazzo sono fortunatamente molto numerose, in ragione dei molteplici e differenti interessi che ruotavano intorno alla struttura. A causa delle differenti dominazioni straniere che si sono avvicendate nel territorio mantovano nel periodo preunitario, le fonti relative a questo lasso di tempo sono conservate in Italia, Francia ed Austria. In ragione di differenti tendenze all'accentramento amministrativo durante il periodo unitario, le fonti più recenti sono variamente distribuite sul territorio nazionale: la sede principale di conservazione è Mantova, a cui si affiancano - per quanto riguarda gli archivi pubblici - Verona, Venezia, Brescia, Milano, Torino e Roma. Per ciò che attiene gli archivi privati si devono prendere in considerazione anche le località legate alle singole personalità che nel tempo hanno legato le loro sorti alla storia del complesso.

\section{La rilevazione delle fonti documentarie individuate}

Preso atto della complessità delle fonti documentarie, si è cercato di individuare un sistema di censimento delle fonti attinenti. Si è quindi redatta la struttura di una piccola base di dati in forma tabellare, di cui, di seguito, si riporta la scansione:

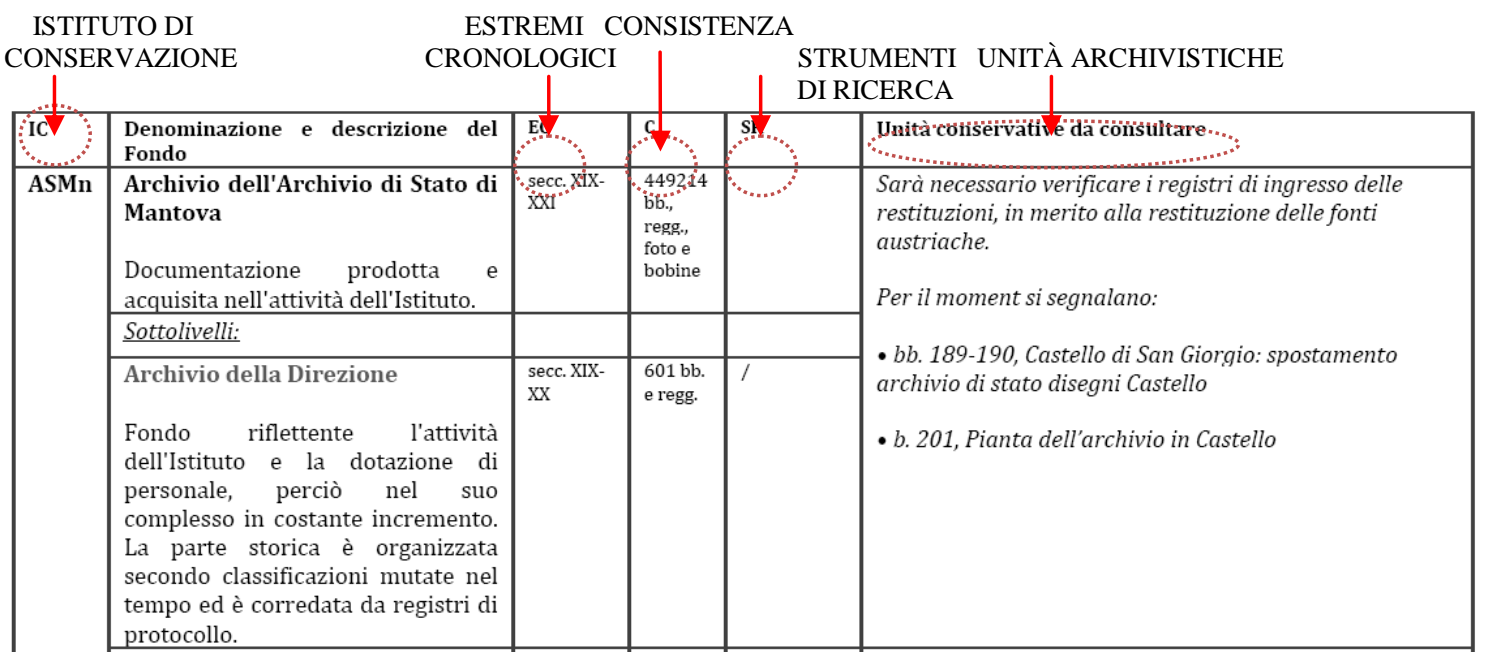

Il medesimo sistema è stato adoperato per compiere lo spoglio dell'Archivio Storico della Soprintendenza per i Beni Storici, Artistici ed Etnoantropologici per le Provincie di Brescia, Cremona e Mantova e la redazione di un primo stato di consistenza del patrimonio documentario in esso contenuto, che ho potuto svolgere durante il 2011 
grazie alla disponibilità del direttore del museo Dottor Stefano L'Occaso, archivio che si è rivelato fondamentale per la ricostruzione degli eventi architettonici del Palazzo Ducale di Mantova.

La scelta della tipologia dei dati da inserire segue questa scansione, che risponde alle esigenze minime di descrizione di un nucleo di fonti:

- Istituto di conservazione (dove si conservano le fonti?);

- Denominazione del fondo (in quale fondo le fonti sono conservate?);

- Estremi cronologici (quale intervallo cronologico ricopre il fondo?);

- Consistenza del fondo (di quanto materiale si tratta? Quali divisioni presenta al suo interno?);

- Strumenti di ricerca (quali strumenti di consultazione sono disponibili? quale grado di descrizione analitica dei contenuti è disponibile?);

- Unità archivistiche (quali "buste” sono da consultare?).

La scansione tipologica dei dati da inserire è stata scelta in ragione dei dati normalmente impiegati nella segnalazione della segnatura di una fonte archivistica e per confronto con quanto proposto nel Sistema Informativo degli Archivi di Stato. Il SIAS è un sistema informativo organizzato dall'Istituto Centrale per gli archivi ed è costituito da una base di dati - in continuo incremento - relativi ai complessi documentari conservati dagli Archivi di Stato italiani, attraverso il quale è possibile compiere ricerche. 


\section{Le schede di spoglio delle unità archivistiche}

Nel procedere allo spoglio delle singole unità archivistiche si è avvertita la necessità di dotarsi di un sistema di registrazione omogenea delle informazioni provenienti dalle singole unità archivistiche consultate, redigendo - come nel caso precedente - la struttura di una piccola base di dati nella tabella di seguito:

\begin{tabular}{|c|c|}
\hline SEGNATURA COMPLETA & ASMN, SCALCHERIA, B. 1 \\
\hline $\begin{array}{l}\text { ISTITUTO DI } \\
\text { CONSERVAZIONE }\end{array}$ & ARCHIVIO DI STATO DI MANTOVA \\
\hline FONDO & ARCHIVIO DELLA SCALCHERIA ED AMMINISTRAZIONE DEL PALAZZO DUCALE DI MANTOVA \\
\hline UNITA' ARCHIVISTICA & B. 001 \\
\hline CONTENUTO & $\begin{array}{l}\text { LA STRUTTURA DELLA BUSTA È COSİ COMPOSTA, DA TRE FASCICOLI NON NUMERATI E SEGNATI } \\
\text { COME: } \\
\text { - RACCOLTA DI GRIDE A STAMPA, 1584-1784 } \\
\text { - SPESE PER FUNERALI DELLA CORTE, 1609-1702 } \\
\text { - CONTI DI CUCINA, 1630-1650 } \\
\text { LA BUSTA CONTIENE PRINCIPALMENTE INFORMAZIONI DI CARATTERE ECONOMICO IN } \\
\text { RELAZIONE ALLA GESTIONE DEGLI APPROVVIGIONAMENTI COMPIUTI DALL'UFFICIO DELLA } \\
\text { SCALCHERIA PER LA CORTE ... }\end{array}$ \\
\hline $\begin{array}{l}\text { PERSONE FISICHE E } \\
\text { GIURIDICHE SEGNALATE: }\end{array}$ & MARGHERITA D’ESTE; VINCENZO I GONZAGA; SCALCHERIA \\
\hline $\begin{array}{l}\text { EDIFICI/AMBIENTI } \\
\text { SEGNALATI: }\end{array}$ & $\begin{array}{l}\text { PALAZZO DuCALE, CORTE VECCHIA (MN0001.B), IN PARTICOLARE LA SALA DELLE } \\
\text { SEGNATURE (MN0001.B.02.28.105) }\end{array}$ \\
\hline
\end{tabular}

\section{Le schede per il regesto e la trascrizione dei documenti}

Dei documenti maggiormente significativi si è riscontrata la necessità di fornire la segnalazione ed il corrispondente regesto e, in alcuni casi, la trascrizione parziale o integrale. Si è proceduto a creare, secondo il sistema descritto in precedenza, sotto forma di tabella, una scheda per il regesto e la trascrizione dei documenti:

\begin{tabular}{|c|c|}
\hline SEGNATURA COMPLETA & $\begin{array}{l}\text { ASMn, SCALCHERIA, B. 1. 1613, LUGLIO 30, MANTOVA. LETTERA DI MARGHERITA D’ESTE A } \\
\text { VINCENZO I GONZAGA }\end{array}$ \\
\hline REGESTO & $\begin{array}{l}\text { LETTERA DI MARGHERITA D’ESTE A VINCENZO I GONZAGA IN MERITO ALLA DECORAZIONE } \\
\text { DA COMPIERE AL CAMINO DELLA SALA DELLE SEGNATURE NEL PALAZZO Di CORTE }\end{array}$ \\
\hline $\begin{array}{l}\text { ISTITUTO DI } \\
\text { CONSERVAZIONE } \\
\end{array}$ & ARCHIVIO DI STATO DI MANTOVA \\
\hline FONDO & ARCHIVIO DELLA SCALCHERIA ED AMMINISTRAZIONE DEL PALAZZO DUCALE DI MANTOVA \\
\hline UNITA' ARCHIVISTICA & B. 001 \\
\hline TRASCRIZIONE & $\begin{array}{l}\text { “30 JULIO 1613. MESSER VINCENZO VORREI INCONTRARVI AL MEZZODÌ PER DISCUTERE DEL } \\
\text { COLORE IN CUI FAR PINGERE IL CAMINO DELLA SALA DELLE SEGNATURE, [...] VOSTRA } \\
\text { DEVOTA MARGHERITA" }\end{array}$ \\
\hline $\begin{array}{l}\text { PERSONE FISICHE E } \\
\text { GIURIDICHE SEGNALATE: }\end{array}$ & MARGHERITA D’ESTE; VINCENZO I GONZAGA; \\
\hline $\begin{array}{l}\text { EDIFICI/AMBIENTI } \\
\text { SEGNALATI: }\end{array}$ & SALA DELLE SEGNATURE (MN0001.B.02.28.105) \\
\hline
\end{tabular}

I testi riportati in entrambe le tabelle sono a solo titolo esemplificativo, ma corrispondono alla tipologia di dati dei quali è necessario dare conto.

\section{La redazione del sistema relazionare}


La principale interrogazione a cui il nostro sistema informativo deve essere in grado di fornire una risposta consiste nell'individuazione delle fonti che testimoniano la storia di un determinato quartiere o un ambiente del Palazzo. Gli strumenti fino ad ora presentati rappresentano le strutture necessarie alla creazione delle basi di dati, dove si è introdotta una prima relazione non automatizzata, che prevede da parte del compilatore una prima attribuzione di corrispondenza fra contenuto delle unità archivistiche o dei documenti e i luoghi citati. Vogliamo invece che il sistema informativo permetta di individuare in modo automatizzato tutti i documenti relativi a un dato ambiente o quartiere. Per fare questo le tabelle presentate sono state trasformate in tabelle implementabili utilizzando il programma per la creazione di database relazionari Base, in cui è stato possibile predisporre le necessarie relazioni ed interrogazioni che ci si è prefissi di ottenere. Si procederà nella prossima fase ad affinare gli strumenti di ricerca del sistema informativo e di implementarne le basi di dati, al fine di agevolarne un futuro transito in sistemi informativi territoriali come, ad esempio, nel progetto Sicar10 del Ministero per i Beni e le attività culturali.

10 Si veda come introduzione ai temi del progetto BARACCHINI, Clara, Uno strumento informatico per la gestione della storia della tutela, in PIVA, Chiara, SGARBOZZA, Ilaria (a cura di), Il corpo dello stile. Cultura e lettura del restauro nelle esperienze contemporanee, Roma, De Luca Editori, 2005, pp. 339-341. 


\section{Riferimenti bibliografici}

ATZENI, Paolo, CERI, Stefano, PARABOSCHI, Stefano, TORLONE, Riccardo, Basi di dati. Modelli e linguaggi di interrogazione, Milano, McGraw-Hill, 2002.

BALBONI, Laura, CORRADINI, Paolo, Rilievo, diagnosi e monitoraggio del degrado statico della "Trostburg" a Waidbruck (BZ), Tesi di laurea, relatore prof. Alberto Grimoldi, Politecnico di Milano, Facoltà di Architettura, Campus Leonardo, Sede di Mantova, a.a. 2003/2004.

BARACCHINI, Clara, Uno strumento informatico per la gestione della storia della tutela, in PIVA, Chiara, SGARBOZZA, Ilaria (a cura di), Il corpo dello stile. Cultura e lettura del restauro nelle esperienze contemporanee, Roma, De Luca Editori, 2005, pp. 339-341.

BARACCHINI, Clara, BOSCAINO, Ivan, LEVI, Donata, MAFFEI Andrea, «A.R.I.S.T.O.S.: Archivio informatico per la storia della tutela delle opere storico artistiche» in Bollettino d'informazioni del centro Ricerche Informatiche per $i$ Beni Culturali, XII, 2/2002, pp. 57-81.

CARUCCI, Paola, Introduzione generale, in PIANO MORTARI, Maria Teresa, SCANDALIATO CICANI, Isotta (a cura di), Le fonti archivistiche. Catalogo delle guide e degli inventari editi (1861-1991), Roma, Ufficio Centrale per i Beni Archivistici, 1995, pp. 7-23.

D’AMICO, Giuseppe, Progettazione concettuale di sistemi informativi, Milano, Hoepli, 1990.

DEL CURTO, Davide, Das Raumbuch zwischen Bauforschung und Schadenskartierung: Zeiträume und Ursachen für Schäden von Burg Trostburg bei Waidbruck bis Mantua, in DIEKAMP, Anja (Hrsg.), Naturwissenschaft und Denkmalpflege, Innsbruck, Innsbruck University Press, 2007, pp. 79-90. 
FRANCO, Anna, LAIEZZA, Gisella, MAFFEI, Andrea, MOZZO, Marco, VISENTIN, Martina, Un sistema informatico in rete per la storia della tutela e del restauro: ARISTOS per ARTPAST. La fase di assessment: l'attività di coordinamento, URL: < www.artplast.org/fileadmin/template/allegati/giornate_di_studio/ARISTOS_COORD.pdf >.

The society for conservation GIS e-Newsletter : GIS for Cultural Heritage Management in Petra, Jordan, 4, 1/2004,

URL: < www.scgis.org/dos/newsletters/scgs_news_4_1.pdf > .

GUERCIO, Maria, Archivistica informatica, Roma, Carocci, 2001.

HOSSE, Karin, SCHILCHER Matthäus, Temporal GIS for analysis and visualization of cultural heritage,

URL: < cipa.icomos.org/fileadmin/template/doc/Antalya/8.pdf > .

MARESCOTTI, Luca, MASCIONE, Maria, Le tecnologie dell'informazione come strumenti per lo studio e la conservazione del patrimonio edilizio,

URL: < www.mascione.it/pubblico/tecnologie_informazione_2003.pdf > .

MORELli, Cinzia, PLANCES, Elena, SATTALINI, Floriana (a cura di), Primo seminario nazionale sulla catalogazione, Roma, ICCD, 2000.

PETRESCU, Florian, The use of GIS technology in cultural heritage, XXI international CIPA symposium 1-6 october 2007, Athens, Greece,

URL: < www.isprs.org/proceedings/XXXVI/5-C53/FP114.pdf >.

PETZET, Michael, MADER, Gert, Praktische Denkmalpflege, Monaco, Kohlhammer, 1993.

RUI, Li, Urban Heritage Conservation by GIS under Urban Renewal: A Case study of Hankou Historical District in Wuhan, China,

URL: < www.isocarp.net/Data/case_studies/1147.pdf >.

SETTIS, Salvatore, L'illusione dei beni digitali, in SETTIS, Salvatore, Italia S.p.A. L'assalto al patrimonio culturale, Torino, Einaudi, 2002, pp. 67-77. 
VALENTI, Filippo, Nozioni di base per un'archivistica come euristica delle fonti documentarie, in GRANA, Daniela (a cura di), Scritti e lezioni di archivistica, diplomatica e storia istituzionale, Roma, Pubblicazioni degli Archivi di Stato, 2000, pp. 135-224.

VICO, Franco, Costruire il GIS: dati versus processi, in GASTALDO, Guido, PANZERI, Matteo (a cura di), Sistemi Informativi Geografici e Beni Culturali, Torino, Celid, 2000.

VITALI, Stefano, Passato digitale, Milano, Bruno Mondadori, 2004.

Fundamentals of the Geodatabase, NearcGis, ESRI Technical Session, Boston, 2007, URL: < www.northeastarc.org/archive/2007/esritalks/Nearco7GeodatabaseIntro.pdf >.

WICKRAMAGAMAGE, Piyasena, ALAGAN, Ram, GIS as a Tool for Heritage Site Management,

URL: < geic.hq.unu.edu/env/files/Heritage GIS Presentation 28-04-05.pdf >.

ZANNI ROSIELLO, Isabella, Andare in archivio, Bologna, Il Mulino, 1995. 


\section{* L'autore}

Laureatasi in architettura nel 2010, Lisa Valli è dottoranda in conservazione dei Beni Architettonici al Politecnico di Milano; oggetto d'indagine della sua tesi di dottorato è la ricognizione delle fonti che trattano le vicende architettoniche del complesso monumentale del Palazzo Ducale di Mantova dal XIX secolo ad oggi.

URL: < http://studistorici.com/progett/autori/\#Valli >

\section{Per citare questo articolo:}

VALLI, Lisa, «Vecchie abitudini e nuovi strumenti. Un sistema informativo per lo studio delle vicende architettoniche del Palazzo Ducale di Mantova», Diacronie. Studi di Storia Contemporanea: Digital History: la storia nell'era dell'accesso, 29/6/2012,

URL:< http://www.studistorici.com/2012/06/29/valli_numero_10/ >

Diacronie Studi di Storia Contemporanea 3 www.diacronie.it

Risorsa digitale indipendente a carattere storiografico. Uscita trimestrale.

redazione.diacronie@hotmail.it

Comitato di redazione: Marco Abram - Giampaolo Amodei - Jacopo Bassi - Luca Bufarale - Alessandro Cattunar - Alice De Rensis Barbara Galimberti - Deborah Paci - Fausto Pietrancosta - Matteo Tomasoni - Luca Zuccolo

Diritti: gli articoli di Diacronie. Studi di Storia Contemporanea sono pubblicati sotto licenza Creative Commons 2.5

Possono essere riprodotti a patto di non modificarne i contenuti e di non usarli per fini commerciali. La citazione di

estratti è comunque sempre autorizzata, nei limiti previsti dalla legge. 